CRYSTALLOGRAPHIC COMMUNICATIONS

ISSN 2056-9890

\section{Structure cristalline, caractérisation spectroscopique, calcul DFT et analyse de surface Hirshfeld du perchlorate de $p$-toluidinium}

\author{
Meriam Ben Jomaa, ${ }^{a}$ Hammouda Chebbi, ${ }^{a},{ }^{b} *$ Noura Fakhar Bourguiba ${ }^{a}$ et \\ Mohamed Faouzi Zid ${ }^{a}$
}

Reçu le 7 décembre 2017

Accepté le 18 décembre 2017

Edité par L. Van Meervelt, Katholieke Universiteit Leuven, Belgique

Keywords: crystal structure; organic perchlorate; DFT calculations; Hirshfeld surface; fingerprint plots.

CCDC reference: 1540997

Supporting information: this article has supporting information at journals.iucr.org/e

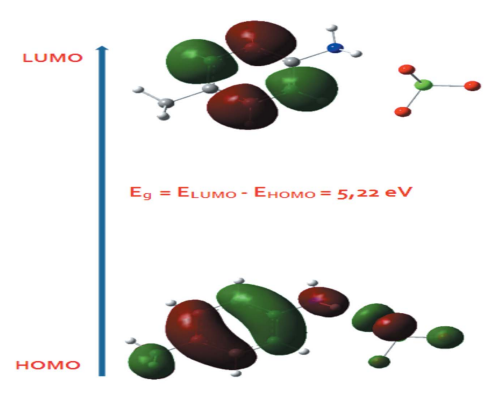

OPEN $\odot$ ACCESS
aUniversité de Tunis El Manar, Faculté des Sciences de Tunis, Laboratoire de Matériaux, Cristallochimie et Thermodynamique Appliquée, 2092 El Manar II, Tunis, Tunisia, et ${ }^{\mathbf{b}}$ Institut Préparatoire aux Etudes d'Ingénieurs de Tunis, Rue Jawaher Lel Nehru, 1089 Montfleury, Tunis, Tunisia. *Correspondence e-mail: chebhamouda@yahoo.fr

The synthesis of $p$-toluidinium perchlorate (systematic name: 4-methylanilinium perchlorate), $\mathrm{C}_{7} \mathrm{H}_{10} \mathrm{~N}^{+} \cdot \mathrm{ClO}_{4}{ }^{-}$, was carried out from an aqueous reaction of perchloric acid with $p$-toluidine. This compound was characterized by powder $\mathrm{XRD}, \mathrm{IR}$ and UV-Vis spectroscopy. The structure was further confirmed by a single-crystal X-ray diffraction study. The crystal structure is formed by a succession of two-dimensional molecular layers consisting of perchlorate anions and organic cations parallel to the (100) plane and located at $x=2 n+\frac{1}{2}(n \in Z)$. Each mixed layer is formed by infinite chains $\left\{\mathrm{C}_{7} \mathrm{H}_{10} \mathrm{~N}^{+} \cdot \mathrm{ClO}_{4}{ }^{-}\right\}_{n}$ parallel to the [010] direction and developing along the $c$ axis, generating $R_{2}^{4}(8), R_{2}^{2}(4)$ and $R_{4}^{4}(12)$ graph-set motifs. The results of a theoretical study using the DFT method at the B3LYP/6-311++G(d,p) level are in good agreement with the experimental data. Hirshfeld surface and fingerprint plots reveal that the structure is dominated by $\mathrm{O} \cdots \mathrm{H} / \mathrm{H} \cdots \mathrm{O}(54.2 \%), \mathrm{H} \cdots \mathrm{H}(26.9 \%)$ and $\mathrm{C}-\mathrm{H} \cdots \pi(14.3 \%)$ contacts. The studied crystal was refined as a two-component twin

\section{Contexte chimique}

Les matériaux hybrides ont fait l'objet de nombreux travaux de recherche du fait qu'ils rassemblent les propriétés des molécules organiques et des composés inorganiques. Cette symbiose entre deux types de chimie trop longtemps considérés comme antagonistes s'hybrident à merveille et libèrent des propriétés complètement nouvelles, et ouvre un vaste champ d'investigations pour le chimiste.

Selon cette approche, les composés hybrides à base de perchlorates ont été particulièrement étudiés du fait des propriétés physiques intéressantes qu'ils présentent comme la ferroélectricité [perchlorate de pyridinium (Czarnecki et al., 1994), perchlorate de pyridin-4-ylméthanaminium (Cui et al., 2016), perchlorate de guanidinium (Drozd \& Dudzic, 2013)] et l'optique non linéaire [perchlorate d'anilinium (Vivek et al., 2015), perchlorate de $p$-nitroanilinium (Bouchouit et al., 2008), perchlorate de L-leucinium (Marchewka \& Drozd, 2013)].

Dans ce contexte, nous avons tenté d'explorer les systèmes $A\left[\mathrm{ClO}_{4}\right]$ ( $A$ : cation organique dérivant de l'anilinium), tel que de s'initier aux différentes techniques de synthèse, de caractérisation et d'analyse structurale ainsi que du calcul théorique DFT. Dans ce travail nous presontons la synthèse, les caractérisations spectroscopiques IR et UV-visible ainsi que la DRX sur poudre, la structure cristalline, le calcul DFT et 
l'analyse de surface Hirshfeld d'un nouveau matériau hybride à base de perchlorate et $p$-toluidinium de formulation $\left(\mathrm{C}_{7} \mathrm{H}_{10} \mathrm{~N}\right)\left[\mathrm{ClO}_{4}\right](\mathrm{I})$.

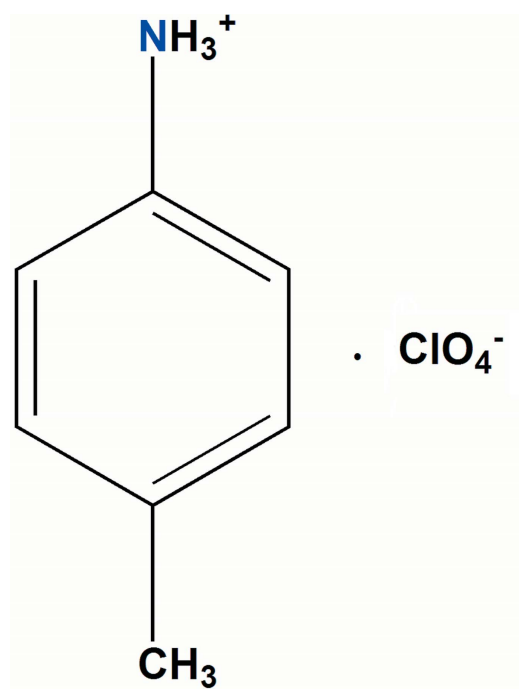

\section{Analyse structurale}

L'unité asymétrique du composé $\left(\mathrm{C}_{7} \mathrm{H}_{10} \mathrm{~N}\right)\left[\mathrm{ClO}_{4}\right]$ est formée d'un anion perchlorate $\left[\mathrm{ClO}_{4}\right]^{-}$et d'un cation organique $\left(\mathrm{C}_{7} \mathrm{H}_{10} \mathrm{~N}\right)^{+}$(Fig. 1). Tous les atomes occupent des positions générales $(4 e)$ et possèdent des faibles facteurs d'agitation thermique comparables à l'exception de celles des atomes d'oxygène $(\mathrm{O} 1, \mathrm{O} 2, \mathrm{O} 3$ et $\mathrm{O} 4)$ du groupement perchlorate qui sont relativement élevés $\left(U_{\mathrm{eq}}>0,1 \AA^{2}\right)$.

L'environnement de l'atome de chlore Cl1 est tétraédrique avec une déformation considérable. En effet, les longueurs des liaisons $\mathrm{Cl}-\mathrm{O}$ varient de 1,394 (5) à 1,421 (4) ̊. Quant aux angles ils présentent une nette dispersion par rapport à l'angle d'un tétraèdre régulier $\left(109,47^{\circ}\right)$, puisque l'intervalle est compris entre $105,3(2)^{\circ}$ et 111,7 (4) $)^{\circ}$ Ces valeurs sont comparables à celles du même anion associé avec d'autres types de cations (Chebbi et al., 2017; Zhou \& Cai, 2012; Dai, 2008; Janczak \& Perpétuo, 2009; Marchewka et al., 2003; Kapoor et al., 2008; Bendjeddou et al., 2009; Liang, 2010).

Les cations organiques $\left(\mathrm{C}_{7} \mathrm{H}_{10} \mathrm{~N}\right)^{+}$occupent des positions générales et assurent l'équilibre des charges négatives excédentaires portées par les anions $\left[\mathrm{ClO}_{4}\right]^{-}$. La valeur moyenne

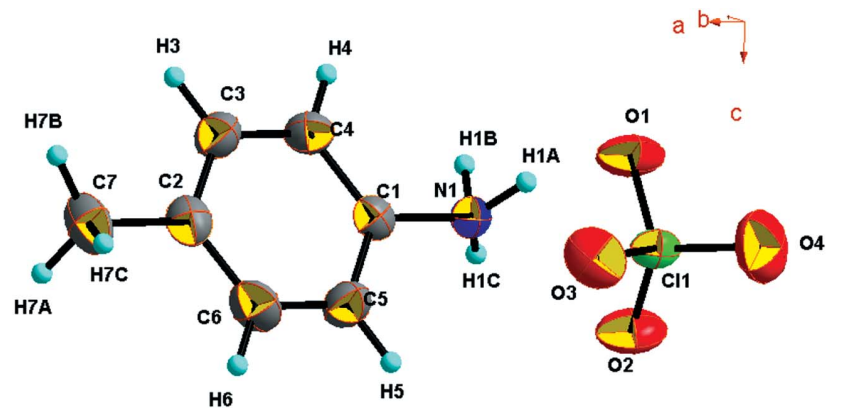

Figure 1

L'unité asymétrique de (I). Les ellipsoïdes d'agitation thermique ont $30 \%$ de probabilité d'existence.
Tableau 1

Géométrie des liaisons hydrogène $\left(\AA{ }^{\circ},{ }^{\circ}\right.$.

\begin{tabular}{lllll}
\hline$D-\mathrm{H} \cdots A$ & $D-\mathrm{H}$ & $\mathrm{H} \cdots A$ & $D \cdots A$ & $D-\mathrm{H} \cdots A$ \\
\hline $\mathrm{N} 1-\mathrm{H} 1 A \cdots \mathrm{O} 1$ & $0.89(1)$ & $2.26(4)$ & $3.011(6)$ & $142(6)$ \\
$\mathrm{N} 1-\mathrm{H} 1 A \cdots 1^{\mathrm{i}}$ & $0.89(1)$ & $2.18(5)$ & $2.891(6)$ & $137(6)$ \\
$\mathrm{N} 1-\mathrm{H} 1 B \cdots 1^{\mathrm{i}}$ & $0.89(1)$ & $2.44(6)$ & $2.891(6)$ & $112(6)$ \\
$\mathrm{N} 1-\mathrm{H} 1 B \cdots \mathrm{O}^{\mathrm{ii}}$ & $0.89(1)$ & $2.42(6)$ & $2.959(7)$ & $120(5)$ \\
$\mathrm{N} 1-\mathrm{H} 1 C \cdots \mathrm{O}{ }^{\mathrm{iii}}$ & $0.89(1)$ & $2.08(3)$ & $2.882(6)$ & $150(6)$ \\
\hline
\end{tabular}

Codes de symétrie: (i) $-x+1,-y,-z+1$; (ii) $x, y+1, z$; (iii) $-x+1, y+\frac{1}{2},-z+\frac{3}{2}$.

de la liaison $\mathrm{C}-\mathrm{C}$ dans le cycle aromatique est égale à

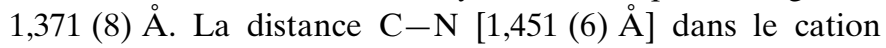
organique $p$-toluidinium est plus longue que la même distance dans le $p$-toluidine neutre [1,386 (4) А; Ploug-Sørenson \& Andersen, 1985], ceci est due à la protonation du groupement amine de la base organique $p$-toluidine. Les angles $\mathrm{C}-\mathrm{C}-\mathrm{C}$ et $\mathrm{C}-\mathrm{C}-\mathrm{N}$ sont compris entre 118,1 (5) et $121,6(6)^{\circ}$, ils ne présentent pas d'anomalies et ils sont comparables à ceux du même cation associé avec d'autres types d'anions (Hosein et al., 2011; Kapoor et al., 2007; Rademeyer, 2005; Xu, 2010; Denne \& Mackay, 1971; Denne et al., 1971; Smirani et al., 2004).

\section{Caractéristiques supramoléculaires}

La cohésion structurale dans l'édifice cristallin est assurée par cinq liaisons hydrogène de type $\mathrm{N}-\mathrm{H}$.. O établies entre les cations et les anions. Les distances et les angles décrivant les liaisons hydrogène sont donnés dans le Tableau 1.

Dans la structure du composé $\left(\mathrm{C}_{7} \mathrm{H}_{10} \mathrm{~N}\right)\left[\mathrm{ClO}_{4}\right]$, les anions et les cations sont reliés par deux liaisons hydrogène $\mathrm{N} 1-$ $\mathrm{H} 1 B \cdots \mathrm{O} 1^{\mathrm{i}}$ [code de symétrie: (i) $-x+1,-y,-z+1$ ] et N1$\mathrm{H} 1 B \cdots \mathrm{O} 3^{\mathrm{ii}}$ [code de symétrie: (ii) $-x, y+1, z$ ] pour former des motifs supramoléculaires $R_{2}^{4}(8)$ autour du centre d'inversion cristallographique situé à $(0,0, z)$. Ces motifs $R_{2}^{4}(8)$ positionnés sur l'unité de translation le long de la direction

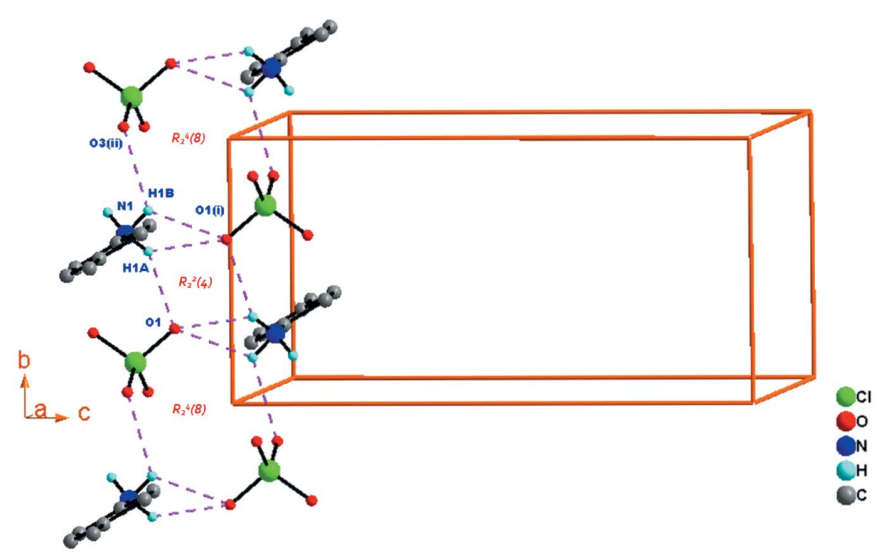

Figure 2

Une partie de la structure cristalline du composé (I) montrant la formation de la chaîne supramoléculaire unidimensionelle, construite par les motifs $R_{2}^{4}(8)$ et $R_{2}^{2}(4)$ s'étendant le long de direction [010]. 


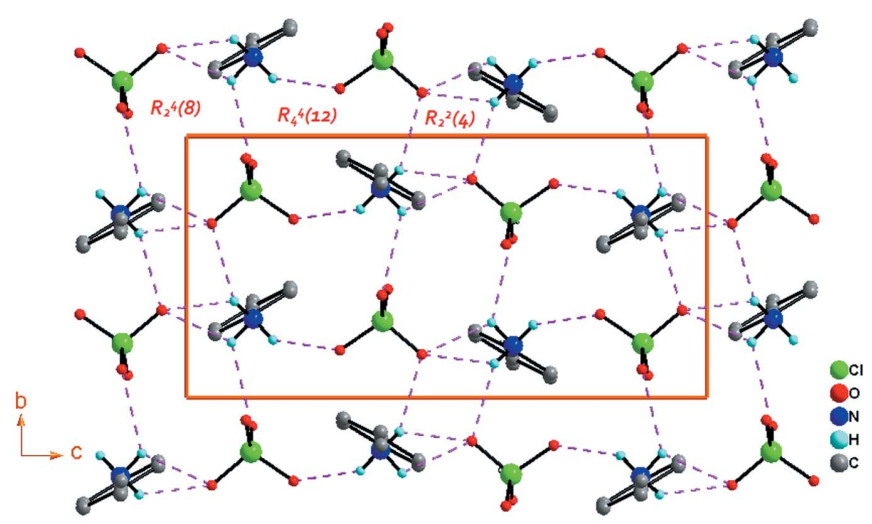

Figure 3

Projection selon la direction [100] d'une partie de la structure cristalline du composé (I) montrant la formation de la couche moléculaire, construite par les motifs $R_{2}^{4}(8), R_{2}^{2}(4)$ et $R_{4}^{4}(12)$, parallèle au plan (100).

[010] sont aussi reliés par deux liaisons hydrogène N1$\mathrm{H} 1 A \cdots \mathrm{O} 1$ et $\mathrm{N} 1-\mathrm{H} 1 A \cdots \mathrm{O} 1^{\mathrm{i}}$ pour former un nouveau motif supramoléculaire supplémentaire de type $R_{2}^{2}(4)$. Ces nouveaux motifs $R_{2}^{2}(4)$ occupent aussi le centre d'inversion cristallographique situé à $\left(0, \frac{1}{2}, z\right)$. La translation infinie des deux motifs $R_{2}^{4}(8)$ et $R_{2}^{2}(4)$ construit une supermolécule sous forme d'une chaîne unidimensionnelle (1D) parallèle à la direction [010] (Fig. 2). Les chaînes adjacentes reliées par l'axe $2_{1}$ sont connectées à travers la liaison hydrogène $\mathrm{N} 1-$ $\mathrm{H} 1 C \cdots \mathrm{O} 2^{\mathrm{iii}}$ [code de symétrie: (iii) $-x+1, y+\frac{1}{2},-z+\frac{3}{2}$ ] située sur l'axe $2_{1}$ le long $\left(0, y, \frac{1}{4}\right)$, en donnant lieu au motif $R_{4}^{4}(12)$ (Fig. 3). Ces connections forment des couches bidimensionnelles d'anions et de cations construites à partir de motifs répétitifs $R_{2}^{4}(8), R_{2}^{2}(4)$ et $R_{4}^{4}(12)$ s'étendant parallèlement au plan (100) (Fig. 3). Une succession de couches moléculaires parallèles au plan (100) et situées à $x=2 n+\frac{1}{2}(n \in Z)$ est représentée dans la Fig. 4.

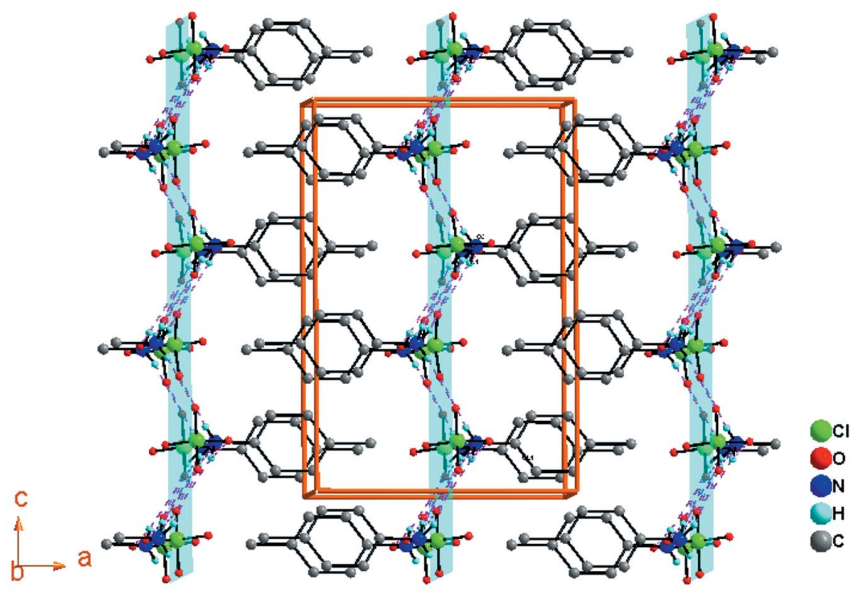

Figure 4

Vue en perspective de la structure cristalline du composé (I) montrant les couches moléculaires parallèles au plan (100).

\section{Caractérisations par DRX sur poudre, IR et UV-Vis}

La diffraction des rayons $\mathrm{X}$ sur poudre, à la longueur d'onde $K_{\alpha}$ du cuivre $(\lambda=1,5405 \AA$ ) du composé (I) a été effectuée au moyen d'un diffractomètre à deux cercles Bruker D8 Advance dans la gamme angulaire $8-70^{\circ}$ en $2 \theta$ avec un pas de comptage de $0,02^{\circ}$ et un temps de comptage de 1 seconde. Les diffractogrammes de poudre exérimental et calculé à l'aide du programme PowderCell (Kraus \& Nolze, 1999) sont présentés dans la Fig. 5. La comparaison des deux difractogrammes montre sans ambiguïté la préparation du composé $\left(\mathrm{C}_{7} \mathrm{H}_{10} \mathrm{~N}\right)\left[\mathrm{ClO}_{4}\right]$ à l'état pur.

En se basant sur des études IR faites sur des composés ayant des similitudes avec le composé $\left(\mathrm{C}_{7} \mathrm{H}_{10} \mathrm{~N}\right)\left[\mathrm{ClO}_{4}\right]$ (Drozd \& Dudzic, 2013; Marchewka \& Drozd, 2013; Ilczyszyn et al., 2002; Anitha et al., 2014; Erdogdu et al., 2012), ainsi que les travaux de Nakamoto (Nakamoto, 1978), nous avons pu attribuer les bandes observées dans le spectre IR représenté dans la Fig. $6 a$ qui a été enregistré entre 400 et $4000 \mathrm{~cm}^{-1}$ en utilisant un spectromètre Perkin-Elmer où l'échantillon est dilué dans la poudre de $\mathrm{KBr}$. Le Tableau 2 regroupe les résultats d'attribution et précise les activités en spectroscopie infrarouge du composé (I).

Les mesures UV-Visible ont été effectuées au moyen d'un spectrophotomètre UNICO 2802 UV/VIS en utilisant une

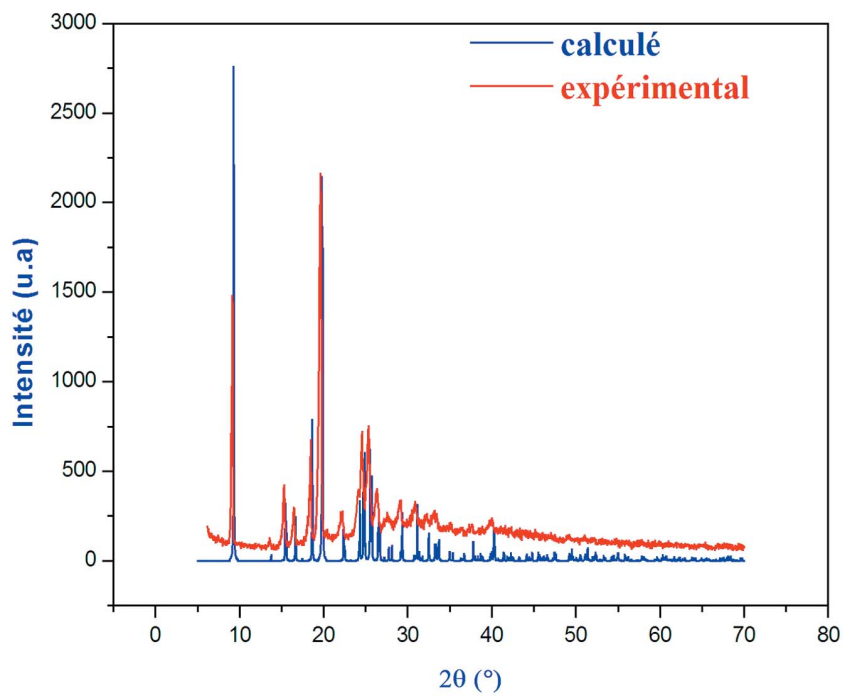

Figure 5

Diffractogrammes de poudre expérimental et calculé du composé (I).

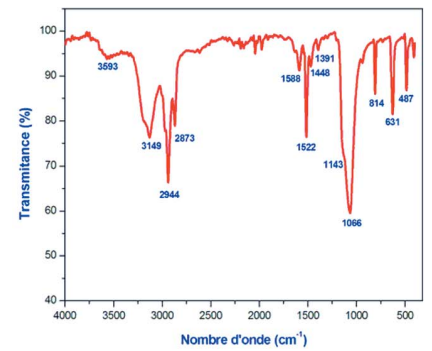

(a)

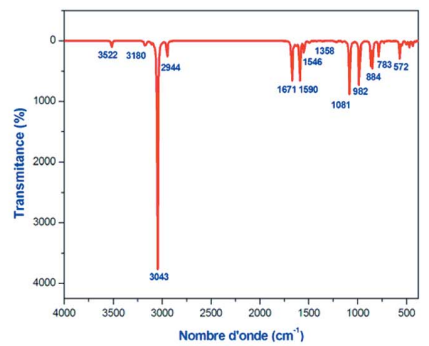

(b)
Figure 6

Spectres infrarouge expérimental (a) et théorique (b) du composé (I). 
Tableau 2

Comparaison de quelques paramètres géométriques $\left(\AA{ }^{\circ},{ }^{\circ}\right)$ observés (X-ray) et calculés (DFT) du composé (I).

\begin{tabular}{llllll}
\hline Paramètre & X-ray & B3LYP/6-311++G(d,p) & Paramètre & X-ray & B3LYP/6-311++G(d,p) \\
\hline Cl1-O1 & $1,416(4)$ & 1,529 & C1-C4 & $1,367(7)$ & 1,394 \\
C11-O2 & $1,421(4)$ & 1,529 & C2-C3 & $1,363(7)$ & 1,396 \\
C11-O3 & $1,396(4)$ & 1,483 & C2-C6 & $1,380(8)$ & 1,401 \\
Cl1-O4 & $1,394(5)$ & 1,464 & C2-C7 & $1,515(7)$ & 1,508 \\
N1-C1 & $1,451(6)$ & 1,469 & C3-C4 & $1,375(7)$ & 1,394 \\
C1-C5 & $1,363(7)$ & 1,391 & C5-C6 & $1,370(8)$ & 1,390 \\
O4-Cl1-O3 & $110,6(3)$ & 112,69 & C3-C2-C6 & $118,1(5)$ & 118,21 \\
O3-Cl1-O2 & $110,1(3)$ & 108,72 & C2-C3-C4 & $121,4(5)$ & 121,06 \\
O1-Cl1-O2 & $105,3(3)$ & 105,94 & C1-C4-C3 & $119,5(5)$ & 119,07 \\
C5-C1-C4 & $120,1(5)$ & 121,54 & C1-C5-C6 & $119,8(5)$ & 118,44 \\
C5-C1-N1 & $119,3(5)$ & 118,67 & C5-C6-C2 & $121,0(5)$ & 121,66 \\
\hline
\end{tabular}

solution diluée de concentration $C=0,019 \mathrm{~mol} \mathrm{~L}^{-1}$. Le spectre UV-Vis obtenu du composé (I) est enregistré dans le domaine 200-800 nm en utilisant l'eau comme solvant (Fig. 7a). Il exhibe une seule bande d'absorption vers $\lambda_{\max }=244 \mathrm{~nm}$ attribuée à la transition $\pi \rightarrow \pi^{*}$ dans le cycle aromatique du cation $p$-toluidinium.

\section{Calcul DFT}

Dans cette étude théorique, la fonctionnelle hybride B3LYP avec la base 6-311++G(d,p) (Becke, 1993) ont été utilisés dans tous les calculs faites par le programme Gaussian 09 (Frisch et al., 2009). L'optimisation de la structure du composé étudié a été réalisée, à partir de la géométrie des rayons $\mathrm{X}$. Une comparaison des résultats expérimentaux avec ceux théoriques (Tableau 2) révèle que la plupart des valeurs calculées de longueurs et d'angles de liaisons sont très proches de celles expérimentales ce qui montre que le choix de la base $6-311++\mathrm{G}(\mathrm{d}, \mathrm{p})$ est convenable pour cette étude théorique, néanmoins la légère différence observée peut être attribuée au environnement de la molécule étudiée, étant isolé en phase gazeuse pour l'étude théorique et soumise à des interactions intermoléculaires à l'état solide dans l'étude expérimentale. Le Spectre IR théorique du composé (I) est représenté dans la Fig. $6 b$ dont les valeurs de fréquences de vibration calculées ont été multiplié par le facteur de correction 0,97 (Tableau 3). Les résultats obtenus avec la méthode DFT [B3LYP/6$311++\mathrm{G}(\mathrm{d}, \mathrm{p})]$ sont en bon accord avec les resultats expérimentaux (Tableau 3). Le spectre UV théorique du composé

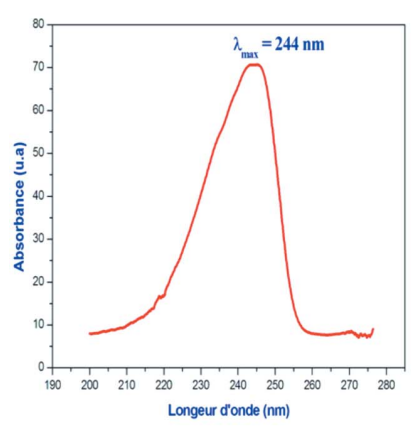

Figure $7 \quad$ (a)

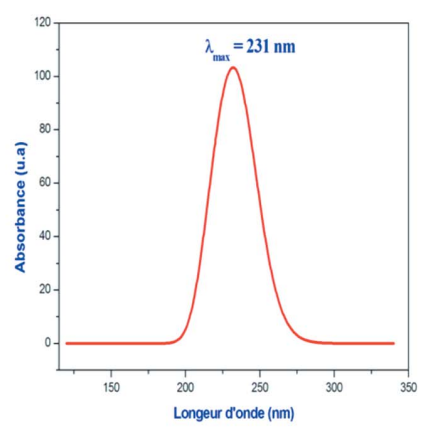

(b)
Spectres UV-Vis expérimental ( $a$ ) et théorique (b) du composé (I).

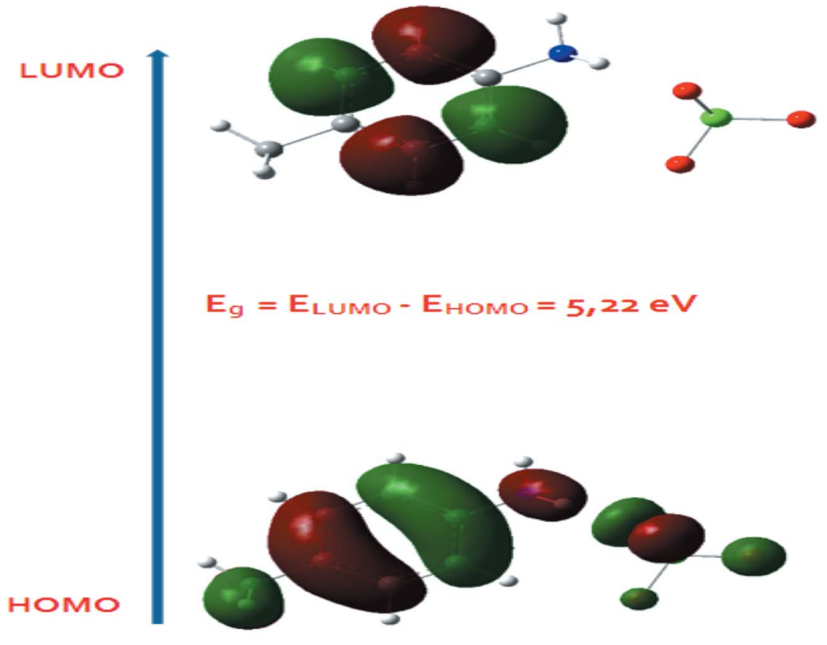

Figure 8

Distribution électronique des orbitales moléculaires LUMO et HOMO du composé (I).

étudié supposé à l'état gazeux a été calculé en utilisant l'approche TD-DFT (Fig. 7b). Il montre une seule bande d'absorption à $\lambda_{\max }$ égale $231 \mathrm{~nm}$ correspondant la transition $\pi-\pi^{*}$. Le calcul théorique nous a permis aussi de calculer les énergies de l'orbitale moléculaire occupée la plus élevée HOMO (Highest Occupied Molecular Orbital) et de l'orbitale moléculaire non occupée la plus basse LUMO (Lowest Unoccupied Molecular Orbital). La distribution électronique des orbitales moléculaires HOMO et LUMO du composé étudié est représentée dans la Fig. 8. L'énergie de gap qui est la différence d'énergie des deux orbitales moléculaires précidentes $\left(E g=E_{\text {LUMO }}-E_{\text {HOMO }}\right)$ vaut $5,22 \mathrm{eV}$.

\section{Analyse de surface Hirshfeld}

Pour avoir un aperçu sur la présence de liaisons hydrogène et des interactions intermoléculaires dans la structure cristalline du composé (I), nous avons utilisé la surface Hirshfeld (Spackman \& McKinnon, 2002; Spackman \& Jayatilaka, 2009) et ses empreintes digitales bidimensionnelles qui sont calculés à l'aide du programme CrystalExplorer (Wolff et al., 2012). Les surfaces Hirshfeld en modes $d_{\text {norm }}$ et $d_{\mathrm{e}}$ sont illustrées dans les Fig. $9 a$ et $9 b$. 
Tableau 3

Comparaison des fréquences expérimentales et calculées $\left(\mathrm{cm}^{-1}\right)$ avec les attributions proposées du composé (I).

\begin{tabular}{lll}
\hline Fréquence expérimentale & Fréquence calculée & Attribution \\
\hline $3593 ; 3149$ & $3416 ; 3084$ & $v_{\text {as }}\left(-\mathrm{NH}_{3}{ }^{+}\right) ; v_{\mathrm{s}}\left(-\mathrm{NH}_{3}{ }^{+}\right)$ \\
$2944 ; 2873$ & $2942 ; 2855$ & $v_{\text {as }}\left(-\mathrm{CH}_{3}\right) ; v_{\mathrm{s}}\left(-\mathrm{CH}_{3}\right)$ \\
$1588 ; 1522$ & $1620 ; 1542$ & $\delta_{\text {as }}\left(-\mathrm{NH}_{3}^{+}\right) ; \delta_{\mathrm{s}}\left(-\mathrm{NH}_{3}^{+}\right)$ \\
1448 & 1499 & $\delta\left(-\mathrm{CH}_{3}\right)$ \\
1391 & 1317 & $v(\mathrm{C}-\mathrm{N})$ \\
$1143 ; 1066$ & $1048 ; 952$ & $v_{\text {as }}\left(\mathrm{ClO}_{4}^{-}\right) ; v_{\mathrm{s}}\left(\mathrm{ClO}_{4}^{-}\right)$ \\
814 & 857 & $v(\mathrm{C}-\mathrm{H})$ \\
$631 ; 487$ & $728 ; 554$ & $\delta_{\text {as }}\left(\mathrm{ClO}_{4}^{-}\right)+\delta_{\mathrm{s}}\left(\mathrm{ClO}_{4}^{-}\right)$ \\
\hline
\end{tabular}

Dans la Fig. $9 a$ les tâches rouges identifiées par l'étiquette 1 correspondent aux contacts rapprochés de type $\mathrm{H} \cdots \mathrm{O}$ qui sont dus aux liaisons hydrogène $\mathrm{N}-\mathrm{H} \cdots \mathrm{O}$. Les zones blanches (étiquette 2) marquent les endroits où la distance séparant les atomes voisins avoisine la somme des rayons de van der Waals des atomes considérés, elles indiquent des interactions de type $\mathrm{H} \cdots \mathrm{H}$. Les zones bleues illustrent les domaines où les atomes voisins sont trop éloignés pour interagir entre eux.

La surface Hirshfeld illustrée dans la Fig. $9 b$ est construite en employant $d_{\mathrm{e}}$ comme mode de représentation. Elle montre aussi l'existence des liaisons hydrogène de type $\mathrm{N}-\mathrm{H} \cdots \mathrm{O}$ et des interactions $\mathrm{H} \cdot \cdots \mathrm{H}$ qui sont représentées par des tâches rouges entourées par des couronnes jaunes (étiquette 1) et des tâches jaunes (étiquette 2), respectivement. La couleur jaune indique que l'atome d'hydrogène de ces interactions est situé à l'intérieur de la surface Hirshfeld. L'emploi de la couleur bleue dans la surface identifiée par l'étiquette 3 indique que les contacts rapprochés dans cette région sont absents vu la grande distance intermoléculaire séparant les atomes voisins.

La Fig.10a illustre l'empreinte bidimensionelle de la totalité des contacts contribuant à la surface Hirshfeld représentée en mode $d_{\text {norm }}$. Le graphique exposé dans la Fig. $10 b$ représente les contacts $\mathrm{H} \cdots \mathrm{O} / \mathrm{O} \cdots \mathrm{H}$ entre les atomes d'hydrogène situés à l'intérieur de la surface et les atomes d'oxygène situés à l'extérieur de la surface Hirshfeld et réciproquement. Il est caractérisé par deux pointes symétriques situées en haut et à gauche et en bas à droite avec $d_{\mathrm{e}}+d_{\mathrm{i}}=1,9 \AA$ (étiquettes 1 et 2). Ces données sont caractéristiques des liaisons hydrogène $\mathrm{N}-\mathrm{H}$-. O. Elles ont la contribution la plus importante à la surface Hirshfeld totale (54,2\%).

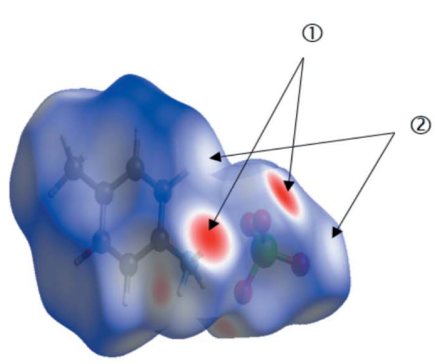

(a)

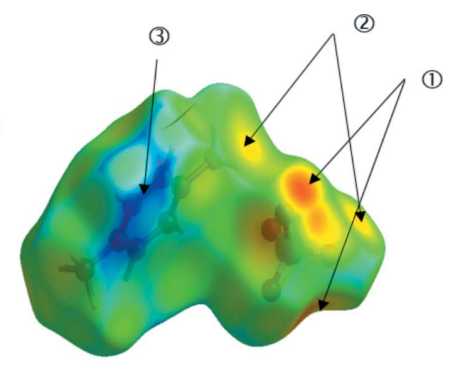

(b)
Figure 9

Surfaces Hirshfeld en modes $d_{\text {norm }}(a)$ et $d_{\mathrm{e}}(b)$ du composé (I).

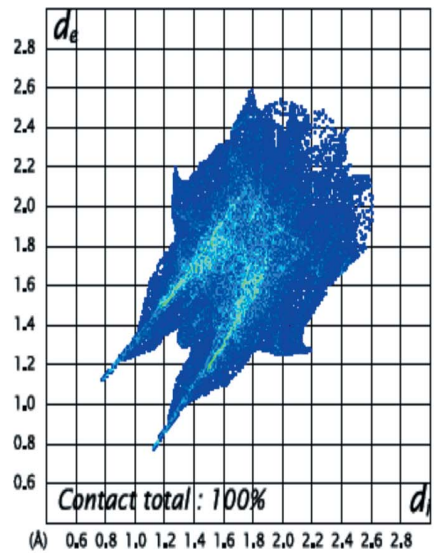

(a)

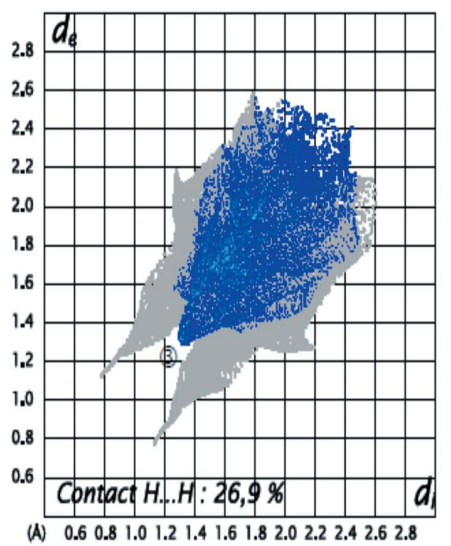

(c)

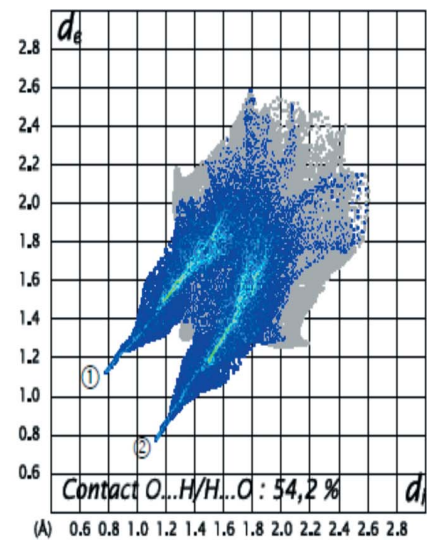

(b)

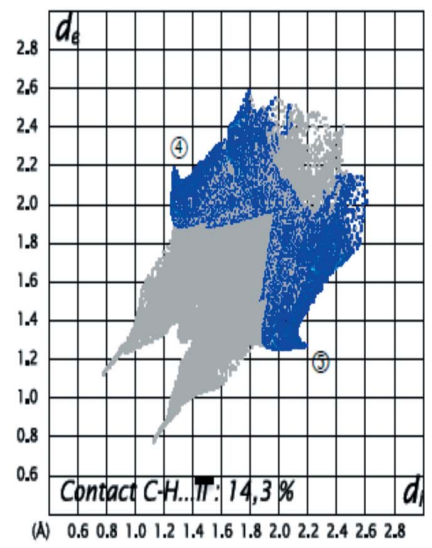

(d)
Figure 10

Empreintes digitales bidimensionelle du composé (I): tous les contacts intermoléculaires $(a)$, contacts $\mathrm{O} \cdots \mathrm{H} / \mathrm{H} \cdots \mathrm{O}(b), \mathrm{H} \cdots \mathrm{H}(c)$ et $\mathrm{C}-\mathrm{H} . . \pi$ (d).

Le graphique représenté dans la Fig. 10c illustre l'empreinte bidimensionelle des points $\left(d_{\mathrm{i}}, d_{\mathrm{e}}\right)$ associés aux atomes d'hydrogène $\left(r_{\mathrm{vdw}}=1,20 \AA\right)$. Il est caractérisé par une extrémité qui pointe vers l'origine et qui correspond à $d_{\mathrm{i}}=d_{\mathrm{e}}=1,3 \AA$ (étiquette 3 ), ce qui révèle la présence des contacts rapprochés $\mathrm{H} \cdots \mathrm{H}$ au sein du composé étudié. Ces contacts $\mathrm{H} \cdots \mathrm{H}$ représentent $26,9 \%$ de la totalité de tous les contacts intermoléculaires.

Le graphique présenté à la Fig. $10 d$ illustre les contacts entre les atomes de carbone situés à l'intérieur de la surface et les atomes d'hydrogène situés à l'extérieur de la surface Hirshfeld et réciproquement. L'analyse de ce graphique montre deux ailes symétriques du cotés gauche et droite (étiquettes 4 et 5). Ces données sont caractéristiques d'une interaction de type $\mathrm{C}-\mathrm{H} \cdots \pi(14,3 \%)$.

La décomposition de l'empreinte digitale bidimensionelle montre aussi d'autres contacts: $\mathrm{O} \cdots \mathrm{O}(2,6 \%), \mathrm{C} \cdots \mathrm{C}(1 \%)$ et $\mathrm{C} \cdots \mathrm{O} / \mathrm{O} \cdots \mathrm{C}(1 \%)$.

\section{Synthèse et cristallisation}

Le composé $\left(\mathrm{C}_{7} \mathrm{H}_{10} \mathrm{~N}\right)\left[\mathrm{ClO}_{4}\right]$ est obtenu en mélangeant dans l'eau, le $p$-toluidine $\mathrm{C}_{7} \mathrm{H}_{9} \mathrm{~N}$ (pureté 99,6\%, Sigma-Aldrich) et 
l'acide perchlorique (pureté 70\%, Merck) selon les proportions molaire 1:1. Après agitation, la solution finale est laissée évaporer à température ambiante. Après cinq jours, des cristaux incolores sous forme de plaquettes commencent à apparaître. Ils ont une taille optimale pour une étude structurale.

\section{Affinement}

Les données cristallographiques, les conditions de la collecte et les résultats de l'affiniment de la structure du composé (I) sont regroupés dans le Tableau 4. Les atomes d'hydrogène liés aux atomes de carbone ont été fixés dans leurs positions géométriques calculés on appliquant les contraintes suivantes: $\mathrm{C}-\mathrm{H}=0,96 \AA$ pour le groupement $-\mathrm{CH}_{3}$ avec $U_{\text {iso }}(\mathrm{H})=$ $1,5 U_{\text {eq }}(\mathrm{C})$ et $\mathrm{C}-\mathrm{H}=0,93 \AA$ pour le groupement $-\mathrm{CH}$ avec $U_{\text {iso }}(\mathrm{H})=1,2 U_{\text {eq }}(\mathrm{C})$. Les atomes d'hydrogène du groupement $-\mathrm{NH}_{3}{ }^{+}$ont été determinés par synthèses de Fourier-différence et en fixant la distance $d_{\mathrm{N}-\mathrm{H}}=0,89 \AA$ avec $U_{\text {iso }}(\mathrm{H})=$ $1,5 U_{\text {éq }}(\mathrm{N})$. Le cristal est maclé par mériédrie avec un angle $\beta=$ $90.41^{\circ}$, émulant un système orthorhombique. Cette macle a été prise en compte en appliquant la matrice de macle $\left[\begin{array}{lllll}1 & 0 & 0,0 & \overline{1} & 0 \text {, }\end{array}\right.$ $\left.\begin{array}{lll}0 & 0 & \overline{1}\end{array}\right]$ durant l'affinement de la structure.

\section{Remerciements}

Soutien financier de la Ministere d'Enseignement Superieur et de la Recherche Scientifique de la Tunisie tient à reconnaitre. Nous remercions vivement Dr Ridha Ben Smail (Institut Préparatoire aux Etudes d'Ingénieurs de Nabeul) pour les discussions fructueuses.

\section{Références}

Anitha, R., Athimoolam, S., Gunasekaran, M. \& Anitha, K. (2014). J. Mol. Struct. 1076, 115-125.

Becke, A. (1993). J. Chem. Phys. 98, 5648-5652.

Bendjeddou, L., Cherouana, A., Hadjadj, N., Dahaoui, S. \& Lecomte, C. (2009). Acta Cryst. E65, o1770-o1771.

Bouchouit, K., Essaidi, Z., Abed, S., Migalska-Zalas, A., Derkowska, B., Benali-cherif, N., Mihaly, M., Meghea, A. \& Sahraoui, B. (2008). Chem. Phys. Lett. 455, 270-274.

Brandenburg, K. (2006). DIAMOND. Crystal Impact GRb, Bonn, Germany.

Chebbi, H., Boumakhla, A., Zid, M. F. \& Guesmi, A. (2017). Acta Cryst. E73, 1453-1457.

Cui, Z., Gao, K., Liu, C., Yin, Y., Fu, D.-W., Cai, H.-L. \& Wu, X. S. (2016). J. Phys. Chem. C, 120, 2925-2931.

Czarnecki, P., Nawrocik, W., Pajak, Z. \& Wasicki, J. (1994). J. Phys. Condens. Matter, 6, 4955-4960.

Dai, J. (2008). Acta Cryst. E64, o2025.

Denne, W. A. \& Mackay, M. F. (1971). J. Cryst. Mol. Struct. 1, 311318.

Denne, W. A., Mathieson, A. M. \& Mackay, M. F. (1971). J. Cryst. Mol. Struct. 1, 55-62.

Drozd, M. \& Dudzic, D. (2013). Spectrochim. Acta Part A, 115, 345356.

Duisenberg, A. J. M. (1992). J. Appl. Cryst. 25, 92-96.

Erdogdu, Y., Drozd, M. \& Marchewka, M. K. (2012). Vib. Spectrosc. 58, 169-180.

Farrugia, L. J. (2012). J. Appl. Cryst. 45, 849-854.

Frisch, M. J., et al. (2009). GAUSSIAN09. Gaussian Inc., Wallingford, CT, USA.
Tableau 4

Détails expérimentaux.

\begin{tabular}{|c|c|}
\hline \multicolumn{2}{|l|}{ Données cristallines } \\
\hline Formule chimique & $\mathrm{C}_{7} \mathrm{H}_{10} \mathrm{~N}^{+} \cdot \mathrm{ClO}_{4}^{-}$ \\
\hline$M_{\mathrm{r}}$ & 207.61 \\
\hline Système cristallin, groupe d'espace & Monoclinique, $P 2_{1} / c$ \\
\hline Température (K) & 293 \\
\hline$a, b, c(\AA)$ & 9,513 (1), 7,196 (5), 14,297 (4) \\
\hline$\beta\left(^{\circ}\right)$ & $90,41(2)$ \\
\hline$V\left(\AA^{6}\right)$ & $978,7(7)$ \\
\hline$Z$ & 4 \\
\hline Type de rayonnement & Мo $K \alpha$ \\
\hline$\mu\left(\mathrm{mm}^{-1}\right)$ & 0,37 \\
\hline Taille du cristal $(\mathrm{mm})$ & $0,6 \times 0,5 \times 0,1$ \\
\hline \multicolumn{2}{|l|}{ Collecte de données } \\
\hline Diffractomètre & Enraf-Nonius CAD-4 \\
\hline Correction d'absorption & $\psi$ scan (North et al., 1968) \\
\hline$T_{\min }, T_{\max }$ & $0,882,0,998$ \\
\hline $\begin{array}{l}\text { Nombre de réflexions mesurées, } \\
\text { indépendantes et observées }[I> \\
2 \sigma(I)] \text { reflections }\end{array}$ & $3216,2128,1160$ \\
\hline$R_{\text {int }}$ & 0,029 \\
\hline$(\sin \theta / \lambda)_{\max }\left(\AA^{-1}\right)$ & 0,638 \\
\hline \multicolumn{2}{|l|}{ Affinement } \\
\hline$R\left[F^{2}>2 \sigma\left(F^{2}\right)\right], w R\left(F^{2}\right), S$ & $0,067,0,212,1,02$ \\
\hline Nombre de réflexions & 2128 \\
\hline Nombre de paramètres & 129 \\
\hline Nombre de restraints & 3 \\
\hline $\begin{array}{l}\text { Traitement des atomes d'hydro- } \\
\text { gène }\end{array}$ & $\begin{array}{l}\text { Atomes d'hydrogène traitées par } \\
\text { un mélange d'affinement indé- } \\
\text { pendant et contraint }\end{array}$ \\
\hline$\Delta \rho_{\max }, \Delta \rho_{\min }\left(\mathrm{e} \AA^{-3}\right)$ & $0,36,-0,21$ \\
\hline
\end{tabular}

Programmes informatiques: CAD-4 EXPRESS (Duisenberg, 1992; Macíček \& Yordanov, 1992), XCAD4 (Harms \& Wocadlo, 1995), SHELXS97 (Sheldrick, 2008), SHELXL2014 (Sheldrick, 2015), DIAMOND (Brandenburg, 2006), WinGX (Farrugia, 2012) et publCIF (Westrip, 2010).

Harms, K. \& Wocadlo, S. (1995). XCAD4. University of Marburg, Germany.

Hosein, A. I., Le Goff, X. F., Ricard, L. \& Caffyn, A. J. M. (2011). Inorg. Chem. 50, 1484-1490.

Ilczyszyn, M., Godzisz, D. \& Ilczyszyn, M. M. (2002). J. Mol. Struct. 611, 103-118.

Janczak, J. \& Perpétuo, G. J. (2009). Solid State Sci. 11, 1576-1581.

Kapoor, I. P. S., Srivastava, P., Singh, G. \& Frohlich, R. (2007). Indian J. Chem. A46, 1283-1288.

Kapoor, I. P. S., Srivastava, P., Singh, G., Singh, U. P. \& Fröhlich, R. (2008). J. Phys. Chem. A, 112, 652-659.

Kraus, W. \& Nolze, G. PowderCell. (1999). Federal Institute for Material Research and Testing, Berlin, Allemagne.

Liang, W.-X. (2010). Acta Cryst. E66, o1575.

Macíček, J. \& Yordanov, A. (1992). J. Appl. Cryst. 25, 73-80.

Marchewka, M. K. \& Drozd, M. (2013). Cent. Eur. J. Chem. 11, 1264 1277.

Marchewka, M. K., Drozd, M. \& Pietraszko, A. (2003). Mater. Sci. Eng. B, 100, 225-233.

Nakamoto, K. (1978). Infrared Spectra of inorganic and coordination compounds, 3eme ed. New York: John Wiley and Sons.

North, A. C. T., Phillips, D. C. \& Mathews, F. S. (1968). Acta Cryst. A24, 351-359.

Ploug-Sørenson, G. \& Andersen, E. K. (1985). Acta Cryst. C41, 613615.

Rademeyer, M. (2005). Acta Cryst. E61, m304-m306.

Sheldrick, G. M. (2008). Acta Cryst. A64, 112-122.

Sheldrick, G. M. (2015). Acta Cryst. C71, 3-8.

Smirani, W., Ben Slimane, A. \& Rzaigui, M. (2004). Z. Kristallogr. New Cryst. Struct. 219, 189-190. 
Spackman, M. A. \& Jayatilaka, D. (2009). CrystEngComm, 11, 1932.

Spackman, M. A. \& McKinnon, J. J. (2002). CrystEngComm, 4, 378392.

Vivek, P., Suvitha, A. \& Murugakoothan, P. (2015). Spectrochim. Acta A Mol. Biomol. Spectrosc. 134, 517-525.
Westrip, S. P. (2010). J. Appl. Cryst. 43, 920-925.

Wolff, S. K., Grimwood, D. J., McKinnon, J. J., Turner, M. J., Jayatilaka, D. \& Spackman, M. A. (2012). Crystal Explorer. University of Western Australia.

Xu, R. (2010). Acta Cryst. E66, o835.

Zhou, B. \& Cai, J. (2012). Acta Cryst. E68, o2113. 


\section{supporting information}

Acta Cryst. (2018). E74, 91-97 [https://doi.org/10.1107/S2056989017018096]

Structure cristalline, caractérisation spectroscopique, calcul DFT et analyse de surface Hirshfeld du perchlorate de $p$-toluidinium

Meriam Ben Jomaa, Hammouda Chebbi, Noura Fakhar Bourguiba et Mohamed Faouzi Zid

Computing details

Data collection: CAD-4 EXPRESS (Duisenberg, 1992; Macíček \& Yordanov, 1992); cell refinement: CAD-4 EXPRESS

(Duisenberg, 1992; Macíček \& Yordanov, 1992); data reduction: XCAD4 (Harms \& Wocadlo, 1995); program(s) used to solve structure: SHELXS97 (Sheldrick, 2008); program(s) used to refine structure: SHELXL2014 (Sheldrick, 2015); molecular graphics: DIAMOND (Brandenburg, 2006); software used to prepare material for publication: WinGX (Farrugia, 2012) and publCIF (Westrip, 2010).

4-Methylanilinium perchlorate

Crystal data

$\mathrm{C}_{7} \mathrm{H}_{10} \mathrm{~N}^{+} \cdot \mathrm{ClO}_{4}^{-}$

$M_{r}=207.61$

Monoclinic, $P 2_{1} / c$

$a=9.513(1) \AA$

$b=7.196(5) \AA$

$c=14.297(4) \AA$

$\beta=90.41(2)^{\circ}$

$V=978.7(7) \AA^{3}$

$Z=4$

Data collection

Enraf-Nonius CAD-4 diffractometer

Radiation source: fine-focus sealed tube

Graphite monochromator

$\omega / 2 \theta$ scans

Absorption correction: $\psi$ scan

(North et al., 1968)

$T_{\min }=0.882, T_{\max }=0.998$

3216 measured reflections

Refinement

Refinement on $F^{2}$

Least-squares matrix: full

$R\left[F^{2}>2 \sigma\left(F^{2}\right)\right]=0.067$

$w R\left(F^{2}\right)=0.212$

$S=1.02$

2128 reflections
$F(000)=432$

$D_{\mathrm{x}}=1.409 \mathrm{Mg} \mathrm{m}^{-3}$

Mo $K \alpha$ radiation, $\lambda=0.71073 \AA$

Cell parameters from 25 reflections

$\theta=10-15^{\circ}$

$\mu=0.37 \mathrm{~mm}^{-1}$

$T=293 \mathrm{~K}$

Plate, colorless

$0.6 \times 0.5 \times 0.1 \mathrm{~mm}$

2128 independent reflections

1160 reflections with $I>2 \sigma(I)$

$R_{\text {int }}=0.029$

$\theta_{\text {max }}=27.0^{\circ}, \theta_{\min }=2.1^{\circ}$

$h=-12 \rightarrow 2$

$k=-1 \rightarrow 9$

$l=-18 \rightarrow 18$

2 standard reflections every 120 reflections intensity decay: $1 \%$

129 parameters

3 restraints

Hydrogen site location: mixed

$\mathrm{H}$ atoms treated by a mixture of independent and constrained refinement 
$w=1 /\left[\sigma^{2}\left(F_{\mathrm{o}}^{2}\right)+(0.085 P)^{2}+1.0514 P\right]$

where $P=\left(F_{\mathrm{o}}^{2}+2 F_{\mathrm{c}}^{2}\right) / 3$

$(\Delta / \sigma)_{\max }<0.001$

$$
\Delta \rho_{\max }=0.36 \mathrm{e} \AA^{-3}
$$

\section{Special details}

Geometry. All esds (except the esd in the dihedral angle between two 1.s. planes) are estimated using the full covariance matrix. The cell esds are taken into account individually in the estimation of esds in distances, angles and torsion angles; correlations between esds in cell parameters are only used when they are defined by crystal symmetry. An approximate (isotropic) treatment of cell esds is used for estimating esds involving 1.s. planes.

Refinement. Refined as a two-component twin

Fractional atomic coordinates and isotropic or equivalent isotropic displacement parameters $\left(\AA^{2}\right)$

\begin{tabular}{lllll}
\hline & $x$ & $y$ & $z$ & $U_{\text {iso }} / U_{\text {eq }}$ \\
\hline C11 & $0.52897(14)$ & $-0.29315(14)$ & $0.62359(8)$ & $0.0642(4)$ \\
O1 & $0.5256(5)$ & $-0.1659(6)$ & $0.5480(2)$ & $0.1049(15)$ \\
O2 & $0.5176(5)$ & $-0.1823(6)$ & $0.7054(3)$ & $0.1103(15)$ \\
O3 & $0.6554(5)$ & $-0.3917(7)$ & $0.6250(4)$ & $0.1262(18)$ \\
O4 & $0.4153(6)$ & $-0.4142(7)$ & $0.6138(4)$ & $0.147(2)$ \\
N1 & $0.6247(5)$ & $0.1992(6)$ & $0.6276(3)$ & $0.0721(11)$ \\
H1A & $0.571(6)$ & $0.132(8)$ & $0.590(4)$ & $0.108^{*}$ \\
H1B & $0.602(7)$ & $0.288(7)$ & $0.587(4)$ & $0.108^{*}$ \\
H1C & $0.582(6)$ & $0.274(7)$ & $0.668(3)$ & $0.108^{*}$ \\
C1 & $0.7767(5)$ & $0.1822(6)$ & $0.6265(3)$ & $0.0589(11)$ \\
C2 & $1.0659(6)$ & $0.1509(8)$ & $0.6266(4)$ & $0.0756(15)$ \\
C3 & $0.9967(6)$ & $0.2379(8)$ & $0.5552(4)$ & $0.0809(15)$ \\
H3 & 1.0480 & 0.2862 & 0.5057 & $0.097^{*}$ \\
C4 & $0.8528(6)$ & $0.2557(8)$ & $0.5547(3)$ & $0.0755(15)$ \\
H4 & 0.8075 & 0.3174 & 0.5059 & $0.091^{*}$ \\
C5 & $0.8435(6)$ & $0.0943(8)$ & $0.6986(4)$ & $0.0827(16)$ \\
H5 & 0.7918 & 0.0449 & 0.7477 & $0.099^{*}$ \\
C6 & $0.9870(6)$ & $0.0790(9)$ & $0.6988(4)$ & $0.0896(18)$ \\
H6 & 1.0321 & 0.0193 & 0.7483 & $0.107^{*}$ \\
C7 & $1.2244(6)$ & $0.1306(12)$ & $0.6272(6)$ & $0.120(3)$ \\
H7A & 1.2640 & 0.2097 & 0.6746 & $0.180^{*}$ \\
H7B & 1.2606 & 0.1656 & 0.5673 & $0.180^{*}$ \\
H7C & 1.2489 & 0.0038 & 0.6402 & $0.180^{*}$ \\
& & & & \\
\hline
\end{tabular}

Atomic displacement parameters $\left(\AA^{2}\right)$

\begin{tabular}{lllllll}
\hline & $U^{11}$ & $U^{22}$ & $U^{33}$ & $U^{12}$ & $U^{13}$ & $U^{23}$ \\
\hline C11 & $0.0915(9)$ & $0.0434(5)$ & $0.0576(6)$ & $0.0046(6)$ & $-0.0020(5)$ & $0.0043(6)$ \\
O1 & $0.179(4)$ & $0.069(2)$ & $0.067(2)$ & $-0.001(3)$ & $-0.005(2)$ & $0.0182(18)$ \\
O2 & $0.163(4)$ & $0.098(3)$ & $0.069(2)$ & $0.022(3)$ & $0.016(2)$ & $-0.016(2)$ \\
O3 & $0.124(4)$ & $0.099(3)$ & $0.155(4)$ & $0.050(3)$ & $-0.014(3)$ & $-0.011(3)$ \\
O4 & $0.144(4)$ & $0.105(4)$ & $0.192(6)$ & $-0.052(4)$ & $-0.026(4)$ & $0.009(4)$ \\
N1 & $0.071(3)$ & $0.059(3)$ & $0.086(3)$ & $0.002(2)$ & $-0.002(2)$ & $-0.014(2)$ \\
C1 & $0.064(3)$ & $0.044(2)$ & $0.068(3)$ & $0.002(2)$ & $-0.005(2)$ & $-0.007(2)$ \\
C2 & $0.070(3)$ & $0.071(3)$ & $0.086(4)$ & $0.004(3)$ & $0.003(3)$ & $-0.020(3)$
\end{tabular}




\begin{tabular}{lllllll} 
C3 & $0.079(4)$ & $0.086(4)$ & $0.079(3)$ & $-0.006(3)$ & $0.014(3)$ & $0.001(3)$ \\
C4 & $0.082(4)$ & $0.076(3)$ & $0.068(3)$ & $0.007(3)$ & $-0.002(2)$ & $0.010(3)$ \\
C5 & $0.085(4)$ & $0.083(4)$ & $0.081(3)$ & $-0.006(3)$ & $0.008(3)$ & $0.015(3)$ \\
C6 & $0.085(4)$ & $0.086(4)$ & $0.097(4)$ & $0.014(3)$ & $-0.021(3)$ & $0.011(3)$ \\
C7 & $0.073(4)$ & $0.144(6)$ & $0.143(6)$ & $0.020(4)$ & $-0.008(4)$ & $-0.050(6)$ \\
\hline
\end{tabular}

Geometric parameters $\left(A,{ }^{\circ}\right)$

\begin{tabular}{|c|c|c|c|}
\hline $\mathrm{C} 11-\mathrm{O} 4$ & $1.394(5)$ & $\mathrm{C} 2-\mathrm{C} 6$ & $1.380(8)$ \\
\hline $\mathrm{Cl1}-\mathrm{O} 3$ & $1.396(4)$ & $\mathrm{C} 2-\mathrm{C} 7$ & $1.515(7)$ \\
\hline $\mathrm{Cl1}-\mathrm{O} 1$ & $1.416(4)$ & $\mathrm{C} 3-\mathrm{C} 4$ & $1.375(7)$ \\
\hline $\mathrm{C} 11-\mathrm{O} 2$ & $1.421(4)$ & $\mathrm{C} 3-\mathrm{H} 3$ & 0.9300 \\
\hline $\mathrm{N} 1-\mathrm{C} 1$ & $1.451(6)$ & $\mathrm{C} 4-\mathrm{H} 4$ & 0.9300 \\
\hline $\mathrm{N} 1-\mathrm{H} 1 \mathrm{~A}$ & $0.885(10)$ & $\mathrm{C} 5-\mathrm{C} 6$ & $1.370(8)$ \\
\hline N1-H1B & $0.887(10)$ & $\mathrm{C} 5-\mathrm{H} 5$ & 0.9300 \\
\hline $\mathrm{N} 1-\mathrm{H} 1 \mathrm{C}$ & $0.885(10)$ & C6-H6 & 0.9300 \\
\hline $\mathrm{C} 1-\mathrm{C} 5$ & $1.363(7)$ & $\mathrm{C} 7-\mathrm{H} 7 \mathrm{~A}$ & 0.9600 \\
\hline $\mathrm{C} 1-\mathrm{C} 4$ & $1.367(7)$ & $\mathrm{C} 7-\mathrm{H} 7 \mathrm{~B}$ & 0.9600 \\
\hline $\mathrm{C} 2-\mathrm{C} 3$ & $1.363(7)$ & $\mathrm{C} 7-\mathrm{H} 7 \mathrm{C}$ & 0.9600 \\
\hline $\mathrm{O} 4-\mathrm{C} 11-\mathrm{O} 3$ & $110.6(3)$ & $\mathrm{C} 2-\mathrm{C} 3-\mathrm{C} 4$ & $121.4(5)$ \\
\hline $\mathrm{O} 4-\mathrm{Cl1}-\mathrm{O} 1$ & $108.3(3)$ & $\mathrm{C} 2-\mathrm{C} 3-\mathrm{H} 3$ & 119.3 \\
\hline $\mathrm{O} 3-\mathrm{Cl1}-\mathrm{O} 1$ & $110.8(3)$ & $\mathrm{C} 4-\mathrm{C} 3-\mathrm{H} 3$ & 119.3 \\
\hline $\mathrm{O} 4-\mathrm{C} 11-\mathrm{O} 2$ & $111.7(4)$ & $\mathrm{C} 1-\mathrm{C} 4-\mathrm{C} 3$ & $119.5(5)$ \\
\hline $\mathrm{O} 3-\mathrm{C} 11-\mathrm{O} 2$ & $110.1(3)$ & $\mathrm{C} 1-\mathrm{C} 4-\mathrm{H} 4$ & 120.3 \\
\hline $\mathrm{O} 1-\mathrm{C} 11-\mathrm{O} 2$ & $105.3(3)$ & $\mathrm{C} 3-\mathrm{C} 4-\mathrm{H} 4$ & 120.3 \\
\hline $\mathrm{C} 1-\mathrm{N} 1-\mathrm{H} 1 \mathrm{~A}$ & $121(4)$ & $\mathrm{C} 1-\mathrm{C} 5-\mathrm{C} 6$ & $119.8(5)$ \\
\hline $\mathrm{C} 1-\mathrm{N} 1-\mathrm{H} 1 \mathrm{~B}$ & $107(4)$ & $\mathrm{C} 1-\mathrm{C} 5-\mathrm{H} 5$ & 120.1 \\
\hline $\mathrm{H} 1 \mathrm{~A}-\mathrm{N} 1-\mathrm{H} 1 \mathrm{~B}$ & $82(5)$ & $\mathrm{C} 6-\mathrm{C} 5-\mathrm{H} 5$ & 120.1 \\
\hline $\mathrm{C} 1-\mathrm{N} 1-\mathrm{H} 1 \mathrm{C}$ & $121(4)$ & $\mathrm{C} 5-\mathrm{C} 6-\mathrm{C} 2$ & $121.0(5)$ \\
\hline $\mathrm{H} 1 \mathrm{~A}-\mathrm{N} 1-\mathrm{H} 1 \mathrm{C}$ & $118(6)$ & $\mathrm{C} 5-\mathrm{C} 6-\mathrm{H} 6$ & 119.5 \\
\hline $\mathrm{H} 1 \mathrm{~B}-\mathrm{N} 1-\mathrm{H} 1 \mathrm{C}$ & $83(5)$ & $\mathrm{C} 2-\mathrm{C} 6-\mathrm{H} 6$ & 119.5 \\
\hline $\mathrm{C} 5-\mathrm{C} 1-\mathrm{C} 4$ & $120.1(5)$ & $\mathrm{C} 2-\mathrm{C} 7-\mathrm{H} 7 \mathrm{~A}$ & 109.5 \\
\hline $\mathrm{C} 5-\mathrm{C} 1-\mathrm{N} 1$ & $119.3(5)$ & $\mathrm{C} 2-\mathrm{C} 7-\mathrm{H} 7 \mathrm{~B}$ & 109.5 \\
\hline $\mathrm{C} 4-\mathrm{C} 1-\mathrm{N} 1$ & $120.5(4)$ & $\mathrm{H} 7 \mathrm{~A}-\mathrm{C} 7-\mathrm{H} 7 \mathrm{~B}$ & 109.5 \\
\hline $\mathrm{C} 3-\mathrm{C} 2-\mathrm{C} 6$ & $118.1(5)$ & $\mathrm{C} 2-\mathrm{C} 7-\mathrm{H} 7 \mathrm{C}$ & 109.5 \\
\hline $\mathrm{C} 3-\mathrm{C} 2-\mathrm{C} 7$ & $121.6(6)$ & $\mathrm{H} 7 \mathrm{~A}-\mathrm{C} 7-\mathrm{H} 7 \mathrm{C}$ & 109.5 \\
\hline $\mathrm{C} 6-\mathrm{C} 2-\mathrm{C} 7$ & $120.4(6)$ & $\mathrm{H} 7 \mathrm{~B}-\mathrm{C} 7-\mathrm{H} 7 \mathrm{C}$ & 109.5 \\
\hline
\end{tabular}

Hydrogen-bond geometry $\left(\AA,{ }^{\circ}\right)$

\begin{tabular}{lllll}
\hline$D-\mathrm{H} \cdots A$ & $D-\mathrm{H}$ & $\mathrm{H} \cdots A$ & $D \cdots A$ & $D-\mathrm{H} \cdots A$ \\
\hline $\mathrm{N} 1-\mathrm{H} 1 A \cdots \mathrm{O} 1$ & $0.89(1)$ & $2.26(4)$ & $3.011(6)$ & $142(6)$ \\
$\mathrm{N} 1-\mathrm{H} 1 A \cdots \mathrm{O} 1^{\mathrm{i}}$ & $0.89(1)$ & $2.18(5)$ & $2.891(6)$ & $137(6)$ \\
$\mathrm{N} 1-\mathrm{H} 1 B \cdots \mathrm{O} 1^{\mathrm{i}}$ & $0.89(1)$ & $2.44(6)$ & $2.891(6)$ & $112(6)$
\end{tabular}


$\mathrm{N} 1-\mathrm{H} 1 B \cdots \mathrm{O} 3^{\text {ii }}$

$\mathrm{N} 1-\mathrm{H} 1 C \cdots \mathrm{O} 2^{\mathrm{iii}}$

Symmetry codes: (i) $-x+1,-y,-z+1$; (ii) $x, y+1, z$; (iii) $-x+1, y+1 / 2,-z+3 / 2$.
$2.42(6)$

$2.08(3)$
$2.959(7)$

$2.882(6)$
$120(5)$

$150(6)$ 